\section{Fibrosis retroperitoneal asociada a neoplasias malignas. Serie de casos clínicos}

\author{
JORGE VEGA $^{1,2,3}$, ALEJANDRO CERIANI $^{1,2}$, DANIELA JENSEN $^{1,2}$
}

\section{Retroperitoneal fibrosis associated with malignancies. Report of eight cases}

Retroperitoneal fibrosis (RPF) is uncommon. Eight percent of cases are associated with malignancies. The pathogenesis of cancer related RPF (MRPF) is unknown. It may be originated from a desmoplastic reaction of the retroperitoneum to the presence of malignant cells, to the action of cytokines secreted by the tumor or a reactive inflammation. MRPF may also be a consequence of antineoplastic therapy (surgery, radiotherapy and chemotherapy). We report eight cases of MRPF associated with breast, cervix, thyroid, kidney and retroperitoneal cancer. In four patients, retroperitoneal tissue biopsy was performed, showing the typical findings of idiopathic RPF. In 6 patients MRPF appeared one to 15 years after cancer diagnosis and in two, it appeared simultaneously. The clinical presentation, course and therapeutic response are described. Six patients were treated with steroids alone and five with steroids associated with colchicine.

(Rev Med Chile 2014; 142: 791-798)

Key words: Neoplasms; Retroperitoneal fibrosis.
'Cátedra de Medicina del Hospital Naval, Escuela de Medicina, Universidad de Valparaíso, Chile. ${ }^{2}$ Servicio de Medicina, Hospital Naval Almirante Nef, Viña del Mar, Chile.

${ }^{3}$ Servicio de Medicina, Sección Nefrología, Hospital Dr. Gustavo Fricke, Viña del Mar, Chile.

Los autores declararon no tener conflictos de intereses.

Recibido el 23 de enero de 2014, aceptado el 16 de mayo de 2014 .

Correspondencia a:

Dr. Jorge Vega Stieb

5 Norte 1035, Viña del Mar.

Teléfono 56-32-2974237

Fax 56-32-2970050

jvegastieb@gmail.com
L a fibrosis retroperitoneal (FRP) se caracteriza por la acumulación de tejido fibroinflamatorio en el retroperitoneo que puede comprimir los uréteres, vasos sanguíneos, linfáticos, intestino y vía biliar, produciendo insuficiencia renal, insuficiencia arterial y venosa, linfedema, obstrucción intestinal y colestasis ${ }^{1,2}$.

La FRP es idiopática en 75\% de los casos. La forma secundaria se ha asociado a: ateromatosis aórtica, enfermedades autoinmunes, vasculitis, asbesto, drogas, enfermedades fibrosantes, radioterapia, quimioterapia, cirugía, hemorragias, infecciones, traumatismos, enfermedades relacionadas con IgG4 y tumores ${ }^{1-5}$. Esta última asociación muy infrecuente $(8-10 \% \text { de las FRP })^{6-13}$.

Esta comunicación describe el modo de presentación clínica en una serie de 8 pacientes con FRP asociada a tumores malignos (FRPM), reunidos en los últimos 5 años en dos hospitales chilenos, su evolución y el tratamiento empleado en ellos.

\section{Casos clínicos}

\section{Caso 1}

Mujer, 61 años. Antecedentes de cáncer cervicouterino diez años antes del ingreso. Consultó por edema de extremidades inferiores (EE.II.). Existía anemia, parámetros inflamatorios elevados e insuficiencia renal asociada a hidronefrosis derecha (Tabla 1). La tomografía computada mostró una masa retroperitoneal (Figura 1). Se instaló un catéter ureteral y se efectuó biopsia quirúrgica del tejido retroperitoneal que mostró FRP. Se prescribió prednisona $30 \mathrm{mg} /$ día y colchicina $1,0 \mathrm{mg} /$ día. Siete meses después apareció hidronefrosis del riñón izquierdo y trombosis de vena cava inferior, instalándose otro catéter ureteral y un filtro en vena cava (Figura 1). La enferma falleció a los 9 meses del diagnóstico de FRP por sepsis urinaria. 


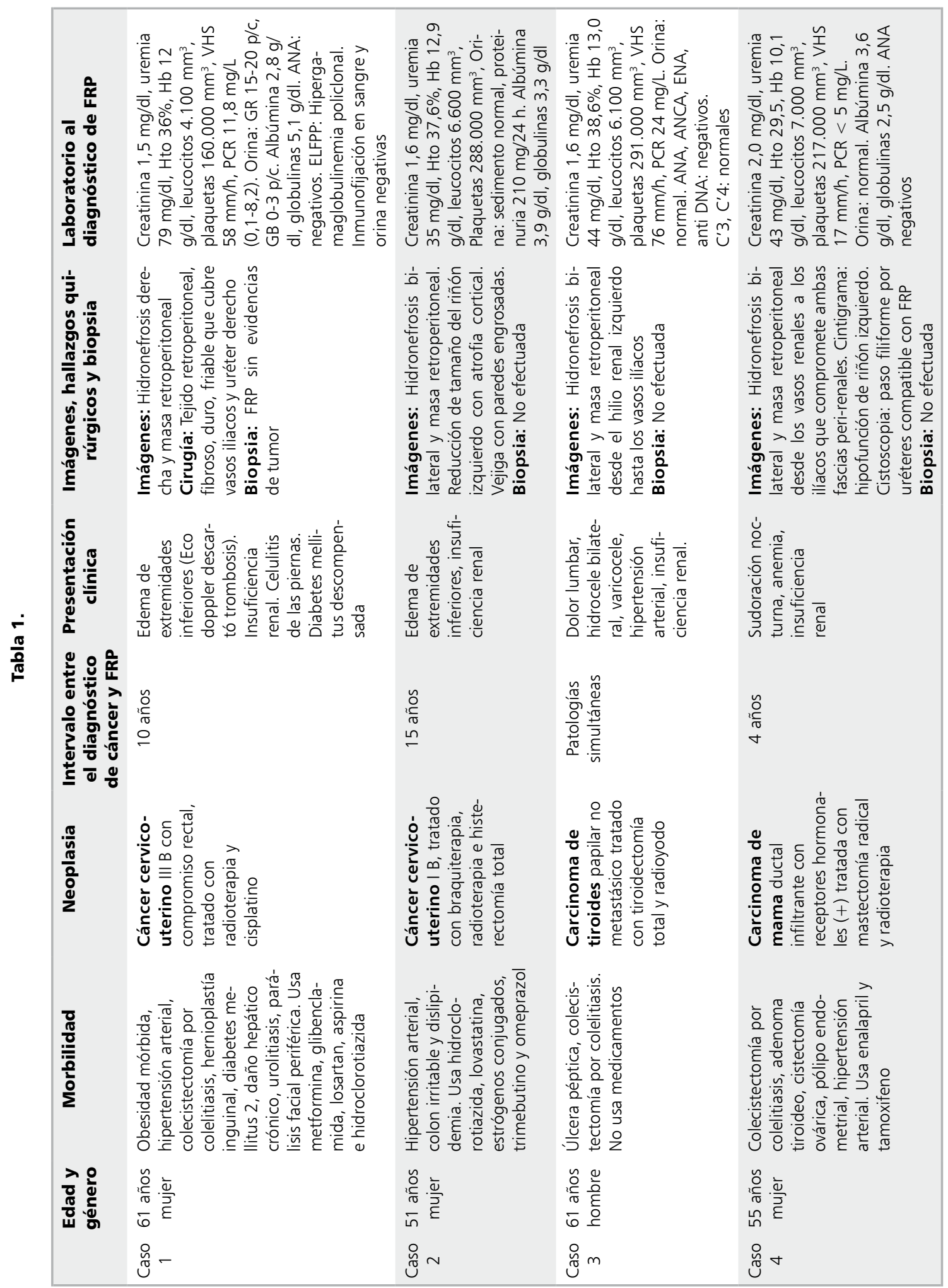




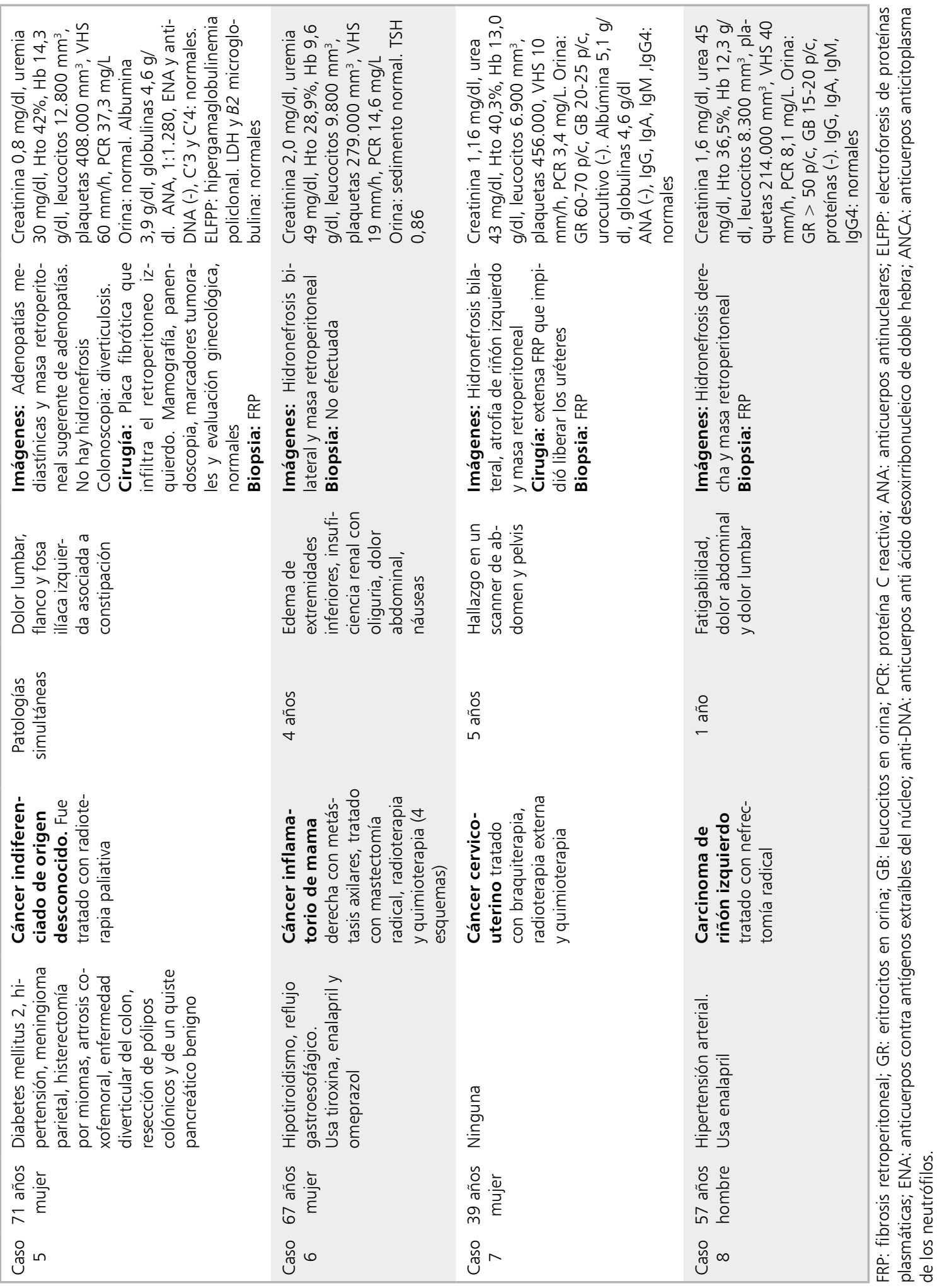



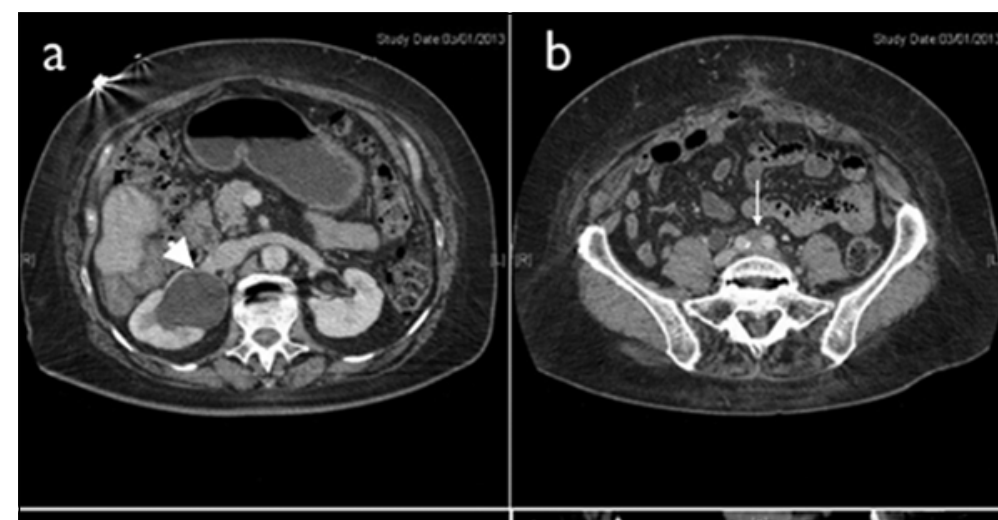

Figura 1. TC con contraste. A: Corte a nivel de las venas renales. Se observa dilatación de la pelvis renal derecha (cabeza de flecha blanca) con indemnidad de la izquierda. B: Se observa a nivel de arterias iliacas la presencia de un tejido que las engloba por su cara interna y posterior (flecha blanca). C: TC sin contraste. Corte axial a nivel de venas
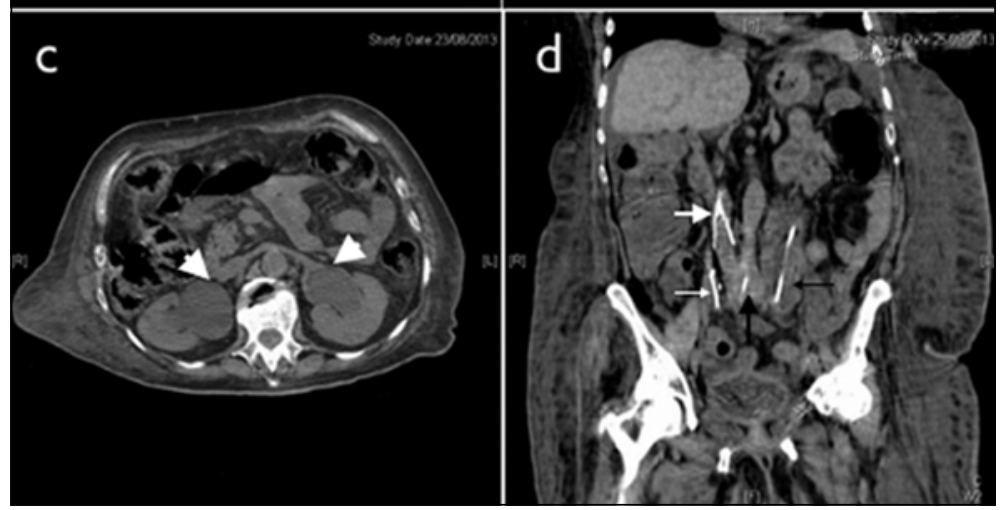
renales, efectuado 8 meses después en que se aprecia dilatación de ambas pelvis renales (cabezas de flechas blancas). D: Corte sagital en que se aprecia desde izquierda a derecha imágenes radioopacas correspondientes a catéter de pigtail en uréter derecho (flecha blanca delgada), filtro en vena cava inferior ( $V$ invertida) (flecha blanca ancha), calcificación en bifurcación aórtica (flecha negra ancha) y catéter de pigtail en el interior de un uréter izquierdo muy dilatado y de paredes engrosadas (flecha negra delgada).

\section{Caso 2}

Mujer, 51 años. Quince años antes del ingreso se trató un cáncer cervicouterino con radioterapia. En un control por hipertensión arterial se encontró edema bilateral de extremidades inferiores, elevación de la creatinina, hidronefrosis bilateral, atrofia e hipofunción del riñón izquierdo y una masa retroperitoneal (Tabla 1). No se efectuó biopsia. Se instalaron catéteres ureterales y la creatinina se normalizó. En los 35 meses siguientes se cambiaron regularmente los catéteres ureterales y la función renal se mantuvo normal, sin evidencias de recidiva del cáncer cervicouterino.

\section{Caso 3}

Hombre, 61 años. A raíz de un cáncer tiroideo fue sometido a una tiroidectomía total y administración de yodo radioactivo. Al mes consultó por fatigabilidad, hidrocele bilateral, varicocele y dolor lumbar, síntomas que sufría desde hacía 9 meses. Existía hipertensión arterial, insuficiencia renal asociada a hidronefrosis bilateral y una masa retroperitoneal. Se instaló un catéter ureteral. La biopsia retroperitoneal reveló una FRP sin células malignas. Se prescribió deflazacort $30 \mathrm{mg} /$ día y colchicina $1,0 \mathrm{mg} /$ día, desapareciendo rápidamente los síntomas. Meses después se retiró el catéter ureteral. $\mathrm{Al}$ año, recibiendo prednisona 5 $\mathrm{mg}$ /día y colchicina $1,0 \mathrm{mg} /$ día, la masa casi había desaparecido, no había hidronefrosis y la función renal era normal. Después de 38 meses, la FRP no ha recurrido.

\section{Caso 4}

Mujer, 55 años. Cuatro años antes se trató por un cáncer de mama. Ingresó por dolor abdominal y sudoración nocturna. Existía insuficiencia renal, hidronefrosis bilateral y una masa retroperitoneal. Se instalaron catéteres ureterales. Seis semanas después se encontraron nódulos peritoneales por metástasis de adenocarcinoma. Se prescribió quimioterapia desapareciendo la hidronefrosis y la masa retroperitoneal. Se retiraron los catéteres ureterales. Falleció 43 meses después del diagnóstico de FRP de una carcinomatosis peritoneal con función renal normal. 


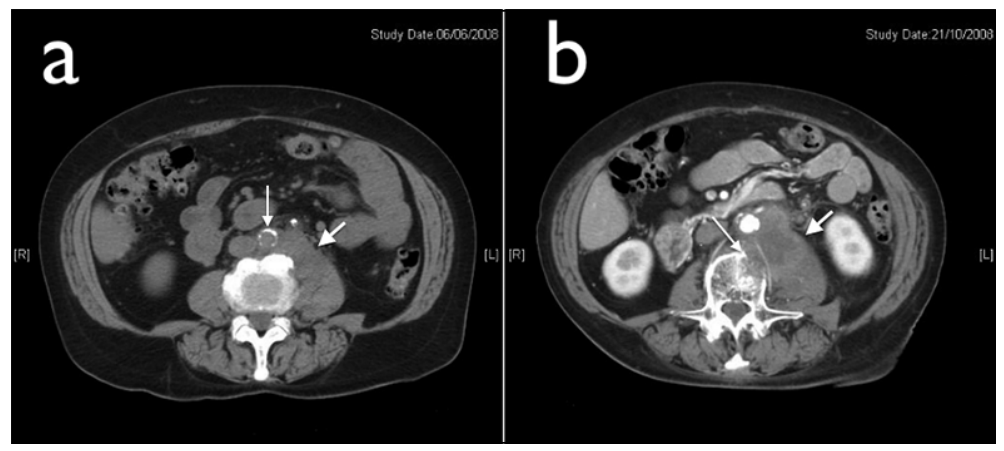

Figura 2. TC sin contraste. A: Se observa tejido retroperitoneal (flecha blanca ancha) que hace cuerpo con el músculo psoas izquierdo y asciende hacia ventral englobando a la aorta (con calcificaciones) (flecha blanca delgada) por su cara externa y posterior. B: TC con contraste efectuado 4 meses después. Se observa crecimiento del tejido retroperitoneal con impregnación heterogénea con el medio de contraste (flecha blanca ancha) e invasión y destrucción de la cortical del cuerpo vertebral adyacente (flecha blanca delgada).

\section{Caso 5}

Mujer, 71 años. Ingresó al hospital por dolor lumbar, flanco y fosa ilíaca izquierda que presentaba desde hacía 6 meses. Se asociaba a constipación. El laboratorio mostró evidencias de inflamación (Tabla 1). Existían adenopatías retroperitoneales y mediastínicas, que hicieron sospechar un linfoma (Figura 2). La biopsia quirúrgica del tejido retroperitoneal diagnosticó una FRP sin células tumorales. Se prescribió prednisona $30 \mathrm{mg} /$ día y colchicina $1,0 \mathrm{mg} /$ día, reduciéndose rápidamente el dolor. Fue hospitalizada nuevamente por reaparición del dolor y baja de peso. Existía aumento de tamaño de la masa retroperitoneal, compromiso del psoas izquierdo y erosión de una vértebra (Figura 2). La biopsia del tejido retroperitoneal reveló células neoplásicas indiferenciadas. Se efectuó radioterapia paliativa. La paciente falleció 10 meses después del diagnóstico de FRP.

\section{Caso 6}

Mujer, 67 años. Cuatro años antes del ingreso se diagnosticó un cáncer de mama derecha. Consultó por dolor abdominal, náuseas, edema bilateral de ambas extremidades inferiores y oliguria. Existía insuficiencia renal, hidronefrosis bilateral y aumento de la densidad del tejido retroperitoneal, compatible con FRP (Tabla 1, Figura 2). Se intentó infructuosamente instalar catéteres ureterales, se efectuó nefrostomía que funcionó defectuosamente e ingresó a hemodiálisis. No se efectuó biopsia retroperitoneal por sus condiciones generales y se prescribió prednisona. La paciente falleció en el hospital a las 6 semanas de su ingreso.

\section{Caso 7}

Mujer, 39 años. Cinco años antes del ingreso se trató un cáncer cervicouterino. En un control rutinario, se encontró hidronefrosis bilateral y atrofia del riñón derecho. Se instalaron catéteres ureterales y se intentó infructuosamente efectuar ureterolisis. La biopsia del tejido retroperitoneal reveló FRP y ausencia de tumor. Se prescribió deflazacort $30 \mathrm{mg} /$ día y colchicina $1,0 \mathrm{mg} /$ día. Al año la masa retroperitoneal había desaparecido y la función renal era normal. A 16 meses del diagnóstico de FRP, la paciente estaba asintomática y sin evidencias de cáncer.

\section{Caso 8}

Hombre, 57 años. Un año antes del ingreso fue sometido a una nefrectomía izquierda por un carcinoma renal. Consultó por fatigabilidad, dolor abdominal y lumbar. Existía hidronefrosis derecha y una masa retroperitoneal. Se instaló un catéter ureteral y se efectuó una biopsia que mostró FRP sin células malignas. Se prescribió prednisona 30 $\mathrm{mg} /$ día y colchicina $1,0 \mathrm{mg}$ /día. Las molestias desaparecieron rápidamente y se normalizó la VHS. Una tomografía computada efectuada 6 meses después mostró desaparición de la hidronefrosis derecha y persistencia de la masa retroperitoneal. $\mathrm{Al}$ año de iniciado el tratamiento reapareció el dolor abdominal y lumbar. Existía un crecimiento de la masa retroperitoneal, adenopatías y metástasis hepáticas. Por recidiva de su cáncer renal ingresó a un protocolo quimioterápico experimental. Diecisiete meses después del diagnóstico de FRP se encontraba en quimioterapia.

\section{Discusión}

La FRPM ha sido descrita en carcinomas de colon, estómago, mama, próstata, páncreas, esófago, riñón, vejiga, uréteres, vesícula biliar, tiroides, cuello uterino, trompa de Falopio, pulmón; 
tumores carcinoides, linfomas, mieloma múltiple y sarcomas ${ }^{7,9,14-29}$. Las asociaciones con tumores benignos son menos frecuentes (angiomiolipomas renales, ganglioneuromas, tumores del aparato yuxtaglomerular $)^{30-32}$. En esta serie los tumores fueron: cervicouterino (3), mama (2), tiroideo, renal y retroperitoneal (Tabla 1 ).

La patogénesis de la FRPM no es clara. Se ha planteado que la fibrosis puede ser secundaria a una reacción dermoplástica a la presencia de células tumorales en el retroperitoneo, producto de citoquinas liberadas por el cáncer o una inflamación reactiva a la presencia del tumor ${ }^{7,9,16,17,20,33,34}$. En los tumores carcinoides se ha relacionado con altas concentraciones sanguíneas de serotonina y neuropéptidos ${ }^{9,35}$. La FRP asociada a un tumor yuxtaglomerular se ha relacionado al sistema renina- angiotensina ${ }^{31}$.

Hay ocasiones en que la FRP precede al diagnóstico del tumor maligno, en otras su diagnóstico es simultáneo y más frecuentemente aparece después que un tumor ha sido tratado. La FRP puede desaparecer después de que el tumor ha sido erradicado ${ }^{18}$. En esta serie a 6 pacientes se les diagnosticó FRP después del cáncer (mediana: 4 años; intervalo 1-15 años) y en 2 ambas patologías fueron simultáneas (Tabla 1).

La FRP se ha asociado a radioterapia. Se ha descrito en seminoma testicular y cánceres de colon, recto, próstata, páncreas y útero ${ }^{6,36,37}$. El intervalo entre la radioterapia y la aparición de la FRP es variable ${ }^{36}$. Cuatro pacientes en nuestra serie recibieron radioterapia (Rö) sobre el abdomen o pelvis, que puede haber favorecido la FRP. El intervalo entre la Rö y el diagnóstico de FRP varió entre 2 y 15 años (mediana: 7,5) (Tabla 1).

La FRP se ha asociado también a quimioterapia. Se han descrito casos en pacientes con linfomas y en cáncer epitelial de ovario ${ }^{38,39}$. En esta serie, 3 pacientes recibieron quimioterapia. El intervalo entre dicha terapia y la FRP fue 4 a 10 años. También la FRP se ha observado después de cirugía del retroperitoneo ${ }^{40}$. Ello ocurrió en 2 pacientes (nefrectomía, histerectomía), 1 y 15 años antes de la FRP.

Radiológicamente la FRPI puede confundirse con linfomas, seminoma testicular, carcinomatosis y sarcomas retroperitoneales ${ }^{30}$. El problema diagnóstico es la diferenciación con un linfoma en un scanner. Mirault et al. propusieron que cuando la FRP se extiende por sobre las arterias renales y desciende bajo la bifurcación aórtica, sin desviación medial de los uréteres, la posibilidad de una FRPM es elevada (sensibilidad: 82\%; especificidad: $83 \%)^{41}$. Otro estudio sugirió que la heterogeneidad en la captación del contraste por la masa retroperitoneal, el desplazamiento de la aorta hacia anterior y la existencia de adenopatías aumentan la probabilidad de linfoma ${ }^{42}$. Se ha recomendado que se efectúe biopsia del tejido retroperitoneal para asegurar el diagnóstico, la que puede efectuarse en forma mínimamente invasiva con una aguja guiada bajo visión radiológica o con cirugía abierta ${ }^{43}$. La apariencia macroscópica del retroperitoneo durante la cirugía frecuentemente no permite distinguir si la FRP es benigna o maligna ${ }^{10}$. La ausencia de células neoplásicas en la biopsia no excluye el diagnóstico de FRPM, por lo que el paciente debe continuar en observación ${ }^{8}$. En esta serie, en 5 pacientes se efectuó una biopsia retroperitoneal y en ninguno se encontró evidencias de tumor. Sin embargo, en dos de ellos la enfermedad neoplásica progresó (casos 5 y 8), por lo cual era probable que junto al tejido retroperitoneal existieran células neoplásicas. Ello se comprobó en uno de los pacientes en una segunda biopsia (caso 5).

Al contrario de la FRPI, en que se ha comunicado una buena respuesta terapéutica a los corticoides, inmunosupresores, tamoxifeno y colchicina $^{3,4,18,44}$, no existen comunicaciones sobre terapia en FRPM, salvo la del tumor asociado ${ }^{18}$. La excepción es la FRPM asociada a un tumor carcinoide ${ }^{7}$. No hay tampoco evidencia que los corticoides sean útiles en la FRPM como lo son en la FRPI.

En esta serie, 6 pacientes se trataron con esteroides y/o colchicina, como se ha comunicado con éxito en pacientes con FRPI, FRP asociada a drogas y pancreatitis autoinmune ${ }^{4-45}$. A la fecha de corte de nuestro estudio (diciembre de 2013) 3 de ellos se encontraban en remisión clínica, 1 tenía evidencias de enfermedad metastásica y 2 habían fallecido (progresión del cáncer, sepsis). La falta de respuesta a los corticoides en una FRPI debe hacer sospechar la presencia de una FRPM, como ocurrió en una de nuestras pacientes (caso 5) y debe buscarse un tumor ${ }^{14}$.

La conducta en la FRPM complicada de insuficiencia renal obstructiva ha sido la desobstrucción mediante catéteres doble J colocados por vía endoscópica o nefrostomía percutánea. 
La mortalidad de los pacientes se produce por el avance del tumor, complicaciones de la quimioterapia, infecciones e insuficiencia renal. En esta serie a la fecha de corte han fallecido 4 pacientes. Las causas de muerte fueron la progresión del cáncer y sepsis urinaria.

En suma, la FRPM puede presentarse años después del tratamiento del tumor y su etiología puede ser variada (idiopática, recurrencia del tumor o complicación de los tratamientos utilizados). La terapia empleada en la FRPI puede ser de utilidad para detener la progresión de la enfermedad u obtener la remisión en algunos pacientes.

\section{Referencias}

1. Vaglio A, Salvarani C, Buzio C. Retroperitoneal fibrosis. Lancet 2006; 367: 241-51.

2. Scheel PJ Jr, Feeley N. Retroperitoneal fibrosis. Rheum Dis Clin N Am 2013; 39: 365-81.

3. Swartz RD. Idiopathic retroperitoneal fibrosis: a review of the pathogenesis and approaches to treatment. Am J Kidney Dis 2009; 54: 546-53.

4. Vega J, Goecke H, Santamarina M. Fibrosis retroperitoneal asociada al uso crónico de ergotamina. Rev Med Chile 2011; 139: 489-94.

5. Kamisawa T, Okamoto A. IgG4-related sclerosing disease. World J Gastroenterol 2008; 14: 3948-55.

6. Peixoto RD, Al-Barrak J, Lim H, Renouf D. Gastroesophageal cancer and retroperitoneal fibrosis: two case reports and review of the literature. World J Gastrointest Oncol 2013; 5: 68-70.

7. Karbasi A, Karbasi-Afshar R, Ahmadi J, Saburi A. Retroperitoneal fibrosis as a result of signet ring cell gastric cancer: a case-based review. J Gastrointest Cancer 2013; 44: 94-7.

8. Hammer ST, Jentzen JM, Lim MS. Anaplastic lymphoma kinase-positive anaplastic large cell lymphoma presenting as retroperitoneal fibrosis. Hum Pathol 2011; 42: 1810-2.

9. Pérez OI, Lizarralde ZL, de Pierola BF, García AL, Crespo CI. Retroperitoneal fibrosis secondary to gastric neoplasia. Actas Urol Esp 2008; 32: 345-7.

10. Gogas J, Markopoulos C, Kouskos E, Gogas H, Kiriakou V. Metastatic retroperitoneal and mediastinal fibrosis as first sign of recurrence of breast cancer. Eur J Surg 2001; 167: 715-8.

11. Sakai D, Murakami M, Kawazoe K, Tsutsumi Y. Ileal carcinoid tumor complicating carcinoid heart disease and secondary retroperitoneal fibrosis. Pathol Int 2000; 50: 404-11.
12. Erton ML, Ilker YN, Ceyhan N, Akdas A. Retroperitoneal fibrosis due to malignancy: a case report. Int Urol Nephrol 1995; 27: 147-50.

13. Armstrong MB, Olson PR, Townsend RN. Gallbladder carcinoma and retroperitoneal fibrosis: a rare combination. J Natl Med Assoc 1989; 81: 1005-6.

14. Yokoyama R, Tazaki R, Morita H, Nishitani H, Ariumi S, Osuga $S$, et al. Retroperitoneal fibrosis in a patient with gastric cancer manifested by lower extremity edema and hidrocele. Intern Med 2012; 51: 2157-60.

15. Milcent K, Franchi-Abella S, Larrar S, Guitton C, Valteau-Couanet D, Koné-Paut I, et al. Retroperitoneal fibrosis and lymphoma in a 15 -year-old boy. Arch Pediatr 2008; 15: 1756-9.

16. Sadhu A, Sen S, Seal S, Sharma SK. Metastasis-an unusual cause of retroperitoneal fibrosis. J Indian Med Assoc 2006; 104: 642-4.

17. Foureur N, Descamps V, Lebrun-Vignes B, Grossin M, Picard-Dahan C, Lecerf V, et al. Retroperitoneal fibrosis secondary to metastatic neoplasm revealed by a leg lymphedema. Ann Dermatol Venereol 2002; 129: 422-3.

18. Templ E, Mostbeck G, Wagner L, Weissel M. Spontaneous healing of retroperitoneal fibrosis after successful therapy of sigmoid carcinoma. Acta Med Austriaca 2000; 27: $168-70$.

19. Dohmen K, Mizukami Y, Tanaka K, Nakamura H, Arase $\mathrm{K}$, Yokogawa Y, et al. Retroperitoneal fibrosis associated with scirrhous gastric cancer. Gastroenterol Jpn 1993; 28: 699-705.

20. Connolly J, Eisner D, Goldman S, Stutzman R, Steiner M. Benign retroperitoneal fibrosis and renal cell carcinoma. J Urol 1993; 149: 1535-7.

21. Fromowitz FB, Miller F. Retroperitoneal fibrosis as host response to papillary renal cell carcinoma. Urology 1991; 38: 259-63.

22. Rivlin ME, McGehee RP, Bower JD. Retroperitoneal fibrosis associated with carcinoma of the cervix: review of the literature. Gynecol Oncol 1991; 41: 95-7.

23. Zinelabidine Y, Chatti N, Jemni L, Boughzala E, Zinelabidine $\mathrm{H}$, Allegue $\mathrm{M}$, et al. Carcinoid tumor of the ilium associated with retroperitoneal fibrosis and right heart involvement. Presse Med 1989; 18: 1847.

24. Beccan G. Multiple myeloma and retroperitoneal fibrosis. Minerva Med 1989; 80: 79-80.

25. Reiner I, Yachia D, Nissim F, Fishelowitz Y. Retroperitoneal fibrosis in association with urothelial tumor. J Urol 1984; 132: 115-6.

26. Bakri YN, Given FT Jr. Retroperitoneal fibrosis complicating stage I B squamous cell carcinoma of the cervix. Gynecol Oncol 1983; 16: 429-35.

27. Martínez Vea A, Darnell A, Quesada E, Ferrer-Roca O, 
Revert L. Malignant retroperitoneal fibrosis associated with pulmonary carcinoma. A review of the literature. Med Clin Barc 1982; 79: 129-32.

28. Anderson KA, Tanagho EA. Fallopian tube carcinoma associated with retroperitoneal fibrosis. J Urol 1981; 125: 119-21.

29. Nasr M, Van Voorhis LW. Retroperitoneal fibrosis associated with pelvic sarcoma. Report of a case. Obstet Gynecol 1970; 36: 606-10.

30. Nakanishi S, Segawa T, Aragaki R, Souda T, Ito N, Kinoshita $\mathrm{H}$, et al. Retroperitoneal ganglioneuroma presenting as a soft tissue mass surrounding the aorta: report of a case. Hinyokika Kiyo 2005; 253-5.

31. Moriya H, Ishida A, Nakabayashi I, Nishiyama Ji, Kobayashi S. Juxtaglomerular cell tumor with retroperitoneal fibrosis and secondary immune-complex glomerulonephritis: a possible contribution of the renin angiotensin system to renal fibrosis. Am J Kidney Dis 1999; 34: e10.

32. Szentgyörgyi E, Kondás J, Diószeghy G. A case of retroperitoneal fibrosis associated with renal angiomyolipoma. Orv Hetil 1991; 132: 1365-7.

33. Esquena S, Abascal JM, Trilla E, De Torres I, Morote J. Retroperitoneal fibrosis simulating local relapse of sarcomatoid renal cell carcinoma. Int Urol Nephrol 2006; 38: 463-5.

34. Yashiro M, Chung YS, Nishimura S, Inoue T, Sowa M. Fibrosis in the peritoneum induced by scirrhous gastric cancer cells may act as "soil" for peritoneal dissemination. Cancer 1996; 77: 1668-75.

35. Dev S, al-Dujaily S, Subbuswamy SG. A case of ureteric obstruction, retroperitoneal fibrosis, and carcinoid tumour. Postgrad Med J 1999; 75: 38-40.

36. Mani R, Niraj A, Sreedhar R. Retroperitoneal fibrosis as long-term complication of radiation treatment for prostate cancer. South Med J 2007; 100: 545-7.

37. Moul JW. Retroperitoneal fibrosis following radiotherapy for stage I testicular seminoma. J Urol 1992; 147: 124-6.

38. Fassina A, Boscolo Berto R, El Mazloum R, Gottardo F, Artibani W. Retroperitoneal fibrosis after chemotherapy. Eur Urol 2007; 51: 270-1.

39. Adab FA, Mould JJ. Carboplatin and retroperitoneal fibrosis. Clin Oncol (R Coll Radiol) 1992; 4: 327-8.

40. Katz R, Golijanin D, Pode D, Shapiro A. Primary and postoperative retroperitoneal fibrosis-experience with 18 cases. Urology 2002; 60: 780-3.

41. Mirault T, Lambert M, Puech P, Argatu D, Renaud A, Duhamel A, et al. Malignant retroperitoneal fibrosis: MRI characteristics in 50 patients. Medicine (Baltimore) 2012; 91: 242-50.

42. Rosenkrantz AB, Spieler B, Seuss CR, Stifelman MD, Kim S. Utility of MRI features for differentiation of retroperitoneal fibrosis and lymphoma. AJR Am J Roentgenol 2012; 199: 118-26.

43. Wan N, Jiao Y. Non-Hodgkin lymphoma mimics retroperitoneal fibrosis. BMJ Case Rep 2013; pii: bcr2013010433.

44. Vega J, Goecke H, Tapia H, Labarca E, Santamarina M, Martínez G. Treatment of idiopathic retroperitoneal fibrosis with colchicine and steroids. A case series. Am J Kidney Dis 2009; 53: 628-37.

45. Vega J, González J, Goecke H, Carrasco A, Santamarina M. Fibrosis retroperitoneal idiopática metacrónicamente asociada a seudotumor de la cabeza de páncreas. Terapia exitosa con corticoides y colchicina. Nefro Plus 2011; 4: 39-44. 